\title{
A Framework for Round the Clock Design and Support
}

\author{
J.P.T. Mo, L. Cirocoo, M. Kovacek \\ CSIRO Manufacturing Science and Technology, Melbourne, \\ Australia \\ Ph: $+61396627759, F x:+61396627851$ \\ Em:jmo@mlb.dmt.csiro.au
}

\begin{abstract}
This paper gives an overview of a project to develop a computer-networked environment which assists designers around the world to work collaboratively. The collaborative environment described here makes use of high bandwidth international networks and is based on the concept of Global Concurrent Engineering (GCE), which enables collaborative work between design teams across time zones and countries. The approach followed in this project is to analyse the process of product design and subsequently develop a working scenario to handle both the interactive and non-interactive activities for global design teams.
\end{abstract}

\section{Keywords}

Global concurrent engineering, computer supported collaborative work, design background information, system interoperability

\section{INTRODUCTION}

The rapid pace of development of information and telecommunication technologies in recent years is globalising the world economy (Nemes and Bertok, 1996) thus placing a demand on many businesses to interact effectively and to market their products globally. In response to this demand for global interaction for doing business, many manufacturing companies, particularly those with global 
operations, are applying the concept of concurrent engineering which shrinks the time to market and incorporates the potential advantages of joint design and engineering efforts for companies with global operations (Sohlenius, 1992). However, the use of GCE requires a new infrastructure and a completely new set of methodologies and tools which are not yet available commercially.

Telecommunications carriers are increasing their investment in high bandwidth technologies for their core networks because it reduces their costs. On the other hand, although there is some investment in high bandwidth technology at the customer interface elements of the network, justification for more commercial investment requires a significant customer application such as GCE. The philosophy of GCE is to allow multiple teams in global companies to work together in a common cyberspace. Likewise, small and medium sized companies can also use GCE to participate in projects of large multi-internationals.

The project described here attempts to address some of the crucial issues relevant to the adoption of GCE by companies for product design. The emphasis of this project is to develop a methodology which is highly flexible and configurable for different user's need (Callaham, 1996). In this paper, we discuss the development of the GCE system from the design to the integration of the GCE system components. The approach in this project is to investigate the global product design process from the user point of view and to identify the activities to be performed by the user. Such an analysis of current scenario provides a direction of the understanding of new scenarios and system development.

\section{CONCEPT OF FRAMEWORK}

The framework described in this paper was developed on the need of the industry partners to mainly support design phase activities. Computer support framework for concurrent engineering applications have been developed by many research groups mostly catered for the need of manufacturing environment (Deitz, 1996; Dong et al, 1996; Dix, 1994). In addressing a specific problem, current methods and tools are inadequate (Frezza et al, 1996). Hence, the strategy in the project is to formulate a number of deliverables which can serve several purposes:

- To generate intermediate improvements on the user scenarios of the project partners.

- To provide experience to participants of the project on the possible latest technologies.

- To establish an experimental environment to investigate the effects of different approaches.

An initial investigation of the current and future working scenarios of design in the global context consists of 3 components: Global Collaborative Environment, Global Information Management and System Interoperation (Figure 1). These components are integrated together by the conceptual GCE process model. Users interact with a system, which is named GCEP to support the design activities of global project team. 


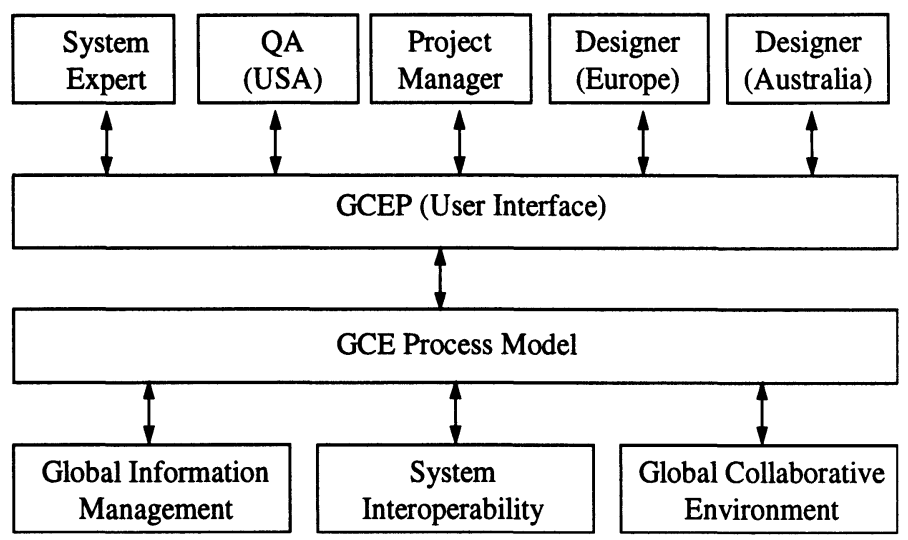

Figure 1 Components of GCEP.

\section{GLOBAL COLLABORATIVE ENVIRONMENT}

The Global Collaborative Environment is a component designed to support simultaneous interactive coordination between distributed work teams. The elements in this component typically include video conferencing, remote meeting room facility, document and application sharing capabilities, multiple site connection and mobile station (Macros, 1996). Due the diversity of computer hardware and software platforms, the environment has been implemented in a system independent way serving Unix, Windows NT and Windows 95 operating systems.

In a point-to-point scenario there are three main types of telemeeting that can be supported with the various tools mentioned above, they are:

- two person, face to face meetings,

- lecturing where there is a single person at one site and many people listening at the other (there may also be a local audience - but the lecturer remains the centre of attention for the duration of the meeting),

- committee meetings where there are multiple participants at both sites.

In a multi-point scenario we have a similar list, but of course we can have combinations of the above, so that a committee meeting could be formed by having single users in a face-to-face environment (desk top or a meeting room), and others in a committee style meeting room. There are also added restrictions/cultural changes in how the collaboration is to be supported (especially in how the video is distributed).

This focus of current work in Global Collaborative Environment to set up various telemeeting tools. Our definition of a telemeeting is any meeting where the participants collaborate from two or more geographically separate locations in real time with a collection of audio, audiovisual and collaborative software tools. 
From the results of previous research, it was found that the audio channel used in a telemeeting must be of the highest possible quality, as even a slight "breaking up" or the presence of too great a delay in the audio can hamper collaboration effectiveness greatly. The usefulness of the video in a telemeeting can vary from irrelevant to critical, depending on the type of interaction required and the possible scenarios for video. The computer based shared workspace will enhance the collaboration greatly as visual ideas can be expressed directly onto a shared work surface. As well as this some collaboration tools allow sharing of standard applications across multiple sites. The implementation of an effective tool/configuration of tools of this type incorporates many different hardware and software combinations. Microsoft NetMeeting is the current defacto conference utility we are testing although any T.120 conference software package should support similar functionality and be able to work with NetMeeting. To use the system it is very similar in practice to software packages such as PictureTel LiveShare Plus and DataBeam Farsite.

There are other tools available for work-station style hardware, but these entry cost for these configurations based on hardware alone have made them less attractive solutions to pursue, although we continue to investigate any developments in this field also. It should be noted that a teleconference with only the audio channel implemented does constitute a telemeeting, but only a necessary subset of one. Without clear and reliable audio, collaboration is greatly impeded. Users with excellent typing skills can begin to bridge the gap on rate and spontaneity of information flow that audio provides. To make telemeetings accessible to a greater number of users a good audio channel will play an important role in facilitating the interaction. For conferencing in a point-to-point scenario, a speaker phone or specialised conference phone at both sites is the optimum solution. Handsets can be used if there is only one participant at any of the sites but this does leave only one hand free to operate the other telemeeting tools.

We have also tried some rather promising Internet based audio tools. These tools have provided good sound quality with a latency of about 1 second, which has been bearable. Unfortunately these tools have only provided point-to-point connectivity and have not allowed conference call connections. Another limitation is that this software does no echo cancellation whatsoever, so headphones must be used, this leads to very restricted single user participation at any of the participating sites (single users are then tethered to their computers via microphones and headphones).

The basic platform for the telemeeting environment is currently a Pentium based PC with monitor, mouse, sound card, speakers (or headphones), microphone and network connectivity; either via a network card or via a $28.8 \mathrm{kbs}$ modem connection. A further enhancement to this set up is to have a large area input loutput surface which when running the appropriate application emulates a whiteboard. We call this tool a LiveBoard, as it not only allows whiteboard interaction at a single site, when connected to other sites the information is displayed at all other sites "live". The way we implement this tool is to have the screen of our base PC rear projected onto a translucent digitising surface. The 
surface then digitises pen presses, drags etc across the image of the screen and moves the mouse pointer accordingly. Video capture can also be introduced into the system either via the cheap parallel port type cameras or via a dedicated video capture card and the latest and greatest broadcast quality video camera.

As one of the aims of the GCEP is to make a platform that can support "any vendor" software application sharing, we also have an X-window server application X-Win so that an application from a unix platform can run on the PC and the $\mathrm{X}$-window is shared to other users via NetMeeting.

At the moment, the software we have selected integrates well with the video display. In fact NetMeeting allows the establishing of an audiovisual connection between 2 parties (in a point-to-point arrangement). Selection possibilities for video equipment lie in the quality of the video capture equipment. There are cheaper "instamatic" focus type cameras, but for a better performance, a fixed focus CCD camera/video capture card combination can give quite good results. Another factor is reduced bandwidth where a simple black and white signal can be selected rather than supporting full colour.

Other peripherals tried so far include a digitising tablet to run in parallel with the mouse. Another peripheral that may be of great use is a flatbed scanner. Another enhancement is to have video incorporated into the PC, either via a parallel port snapshot camera or via a camcorder and video capture card.

\section{GLOBAL INFORMATION MANAGEMENT}

The Global Information Management is designed to support asynchronous coordination for distributed work teams in different time zones. It is based on open architecture to allow information access and control on company Intranet or Internet (Fowler et al, 1996; Love, 1996). It captures, records, retrieve, search and retrieve design intent and background information from distributed information centres. The main emphasis of research in this component is the definition of information objects, conceptual schema and linkages to databases (Prasad, 1996; Kunz et al, 1996).

The purpose for Global Information Management module is to cater for the need of non-interactive sessions between 2 or more geographically separated design groups in different time zones. While the use of Global Collaborative Environment of GCEP is expected to provide an interactive environment for several design groups to work together in a specific time window to communicate, this only occurs in a short period within the 24 hour clock product development cycle. In the other time periods, only people in one time zone will be on-line and all the others will take their rest.

The research to generate a Global Information Management component in GCEP is to provide support to the on-line design group to search, retrieve, view, edit, be alerted, be managed and more importantly, capture design rationale. For example, French (1994) summarised a list of design annotations which are obvious to a group but may not be known to other design groups. To do these, an understanding of the system architecture and elements required to build such a 
system is required. Since most anticipated users are working on hard products design, this work is primarily based on the product model information structure and later on extend to model software products or services using the same principles.

In order to generate the system for particular user requirements, some kind of fundamental methodology should be applied. The methodology will then form the generic basis which allows for future expansion. Basically, the methodology proposed here is to treat all design activities as activities manipulating design objects (Fichman and Kemerer, 1995). The design objects are related to one another (often in multiple relationships). Figure 2 illustrates the structure of a simple product and the attributes in each of the objects.

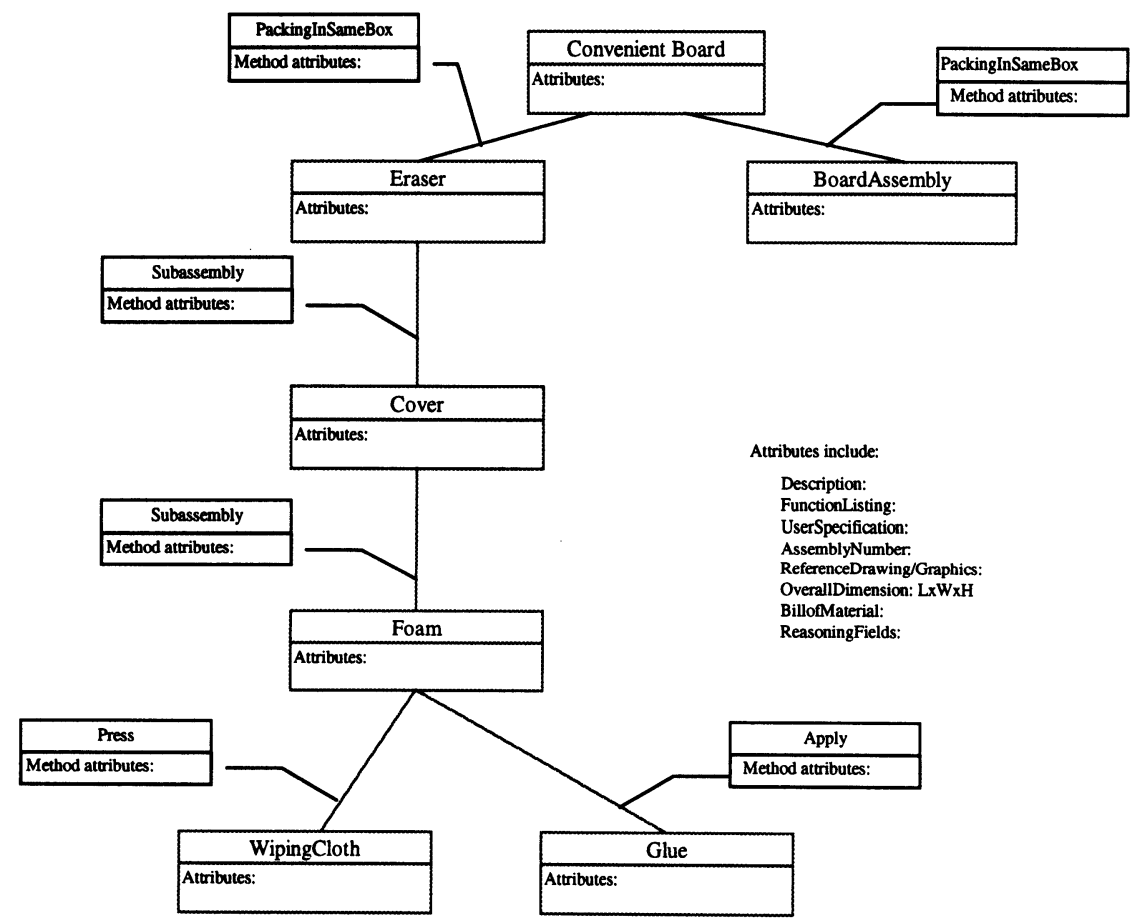

Figure 2 Structure of an object oriented product model.

From this object oriented product model, the system is developed to provide a sequence of activities in which the user will interact with the system to enter information required for other team members. The modelling approach ensures that most background information will be catered for and entered to the system accordingly. During operation, the system will popup with a number of windows as shown in Figure 3. 


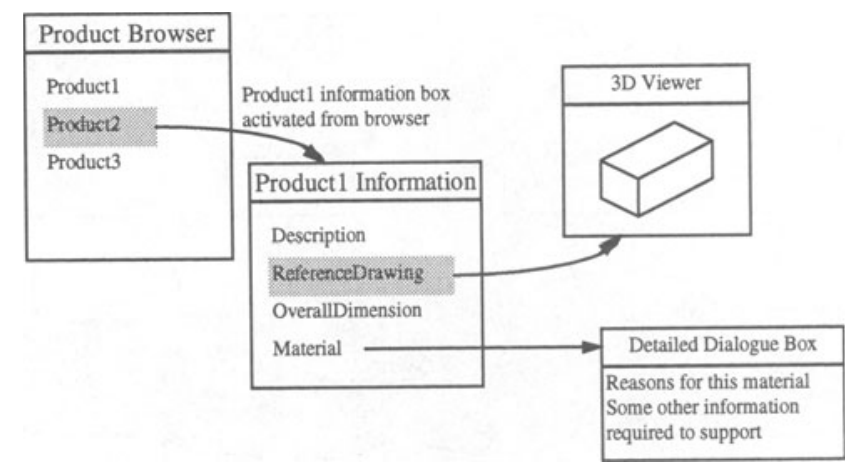

Figure 3 Operation guided by an object oriented product model.

Designers who work on the product will then be able to browse through the information in much the same way as the information has been entered. In addition, when coupled with an activity model, the system will be able to capture the background understanding and provide reasoning deduction from the product model.

\section{SYSTEM INTEROPERABILITY}

The System Interoperation component concentrates on the problems of application interoperability. For example, when one design team produces a product model on a CAD package while the other design team works on the analysis of the product model using finite element method. Similarly, when project management information has to be exchanged between sites but are complicated with the problem of software compatibility. Essentially, research is required is to resolve differences in systems related to heterogeneity, distribution and data fragmentation. In its ultimate form, it requires a flexible and proactive set of software components (often known as middleware) which will provide consistent user and device interfaces to allow applications to work each other over the Internet or Intranet. It will enable structured access to common integration services and information sharing globally. The goal is to create an integrated unique GCE Platform fostering effective and efficient exchange of information over a bandwidth limited global network (Figure 4). 


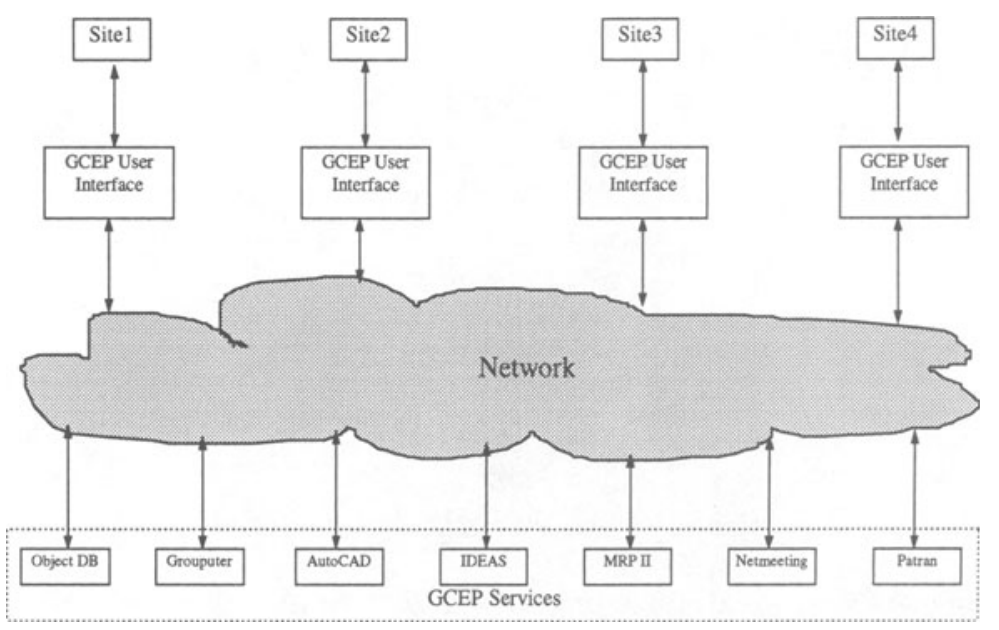

Figure 4 CORBA supported system interoperability.

Part solutions to this component are commercially available including "Message-oriented middleware", "Remote procedure call", "Open database connectivity", "Distributed transaction processing", but all of them are only addressing certain aspects of the problem. The best known technology at the moment which has the potential to be developed into a complete platform is based on the concept of "Object Request Broker" (ORB). The most important development is represented by CORBA specified by the Object Management Group (OMG). This component investigates the application of CORBA technology in the local and international network environment. Wrappers are developed to integrate new applications with existing legacy systems.

\section{GCEP MODELS}

Existing technologies in computer supported collaborative work and network computing are useful only when the users are well aware of the system behaviour and react appropriately. This is a two-sided adaptation. Users need to be trained properly to understand the capabilities and limitations of the GCE Platform. A properly documented system process is required. The researchers and developers of the GCE Platform need to know what design activities they are going to support and hence design the system to support such activities as far as possible. Two major activities are included in this component. Models of the current and future scenarios are created to provide a basis for analysing and improve the ways that the GCEP can be used. The transitions from current to future scenarios are realised through amalgamation and guidance of development of necessary technologies in the other components (Barkan et al, 1993). When the GCEP is developed, the scenarios in the model will be used as the guidance for configuration of systems 
tailored for specific situations and for developing training materials in normal or simulated settings for end users.

The overall aim of this part of the project is to develop and investigate process (including workflow) improvements for round the clock product development, and generalise the findings. To this end it addresses the variants of concurrent software developments (serial and parallel tasks, and multi-site team-based tasks). The approach is to model the product development cycle using IDEF0 as shown in Figure 5.

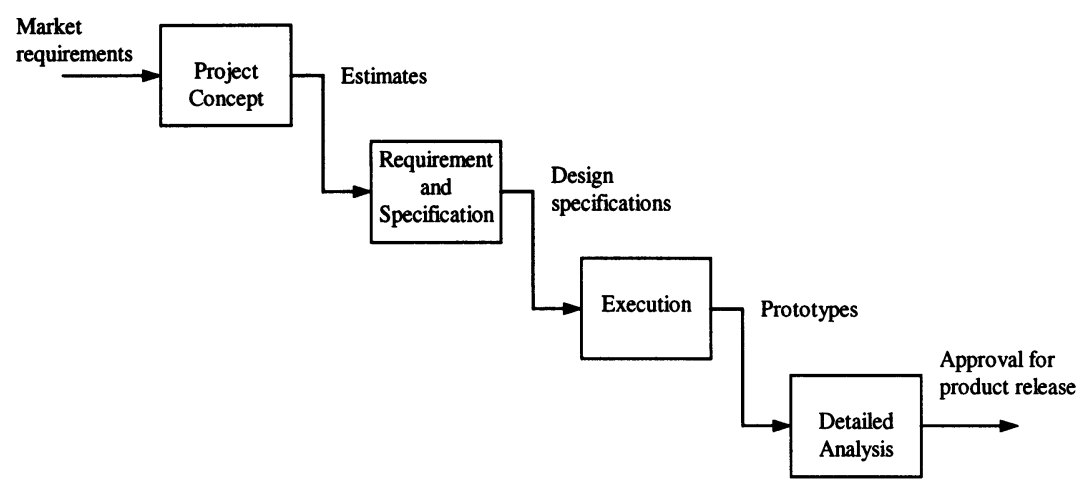

Figure 5 Product design functions.

\subsection{Project concept phase}

The purpose of this is to interpret and clarify customer requirements from different points of view (design, test and industrialisation), analyse whether a product is to be developed, and to make a rough sketch of the ordered system. Also, it establishes a design base, determines whether platform products in generic components are affected, and provides cost, time and resource estimations for a decision to continue. At this stage, a preliminary technical consideration may be given for designing system component. Typical decisions may assess the technical and commercial feasibility of the project.

\subsection{Requirements and specifications phase}

The main purpose of this phase is to analyse the requirements for their feasibility and create a stable system architecture with well-defined and reusable components. This may include activities to evaluate derived and specific component requirements. This may be done from a breakdown of complex system requirements, from different points of view. Other activities may estimate the cost of developing a new component, and to make a rough sketch of the component. During this phase, the project plans are prepared and a comprehensive project organisation is defined for execution. The decision to continue may indicate execution of the project. 


\subsection{Execution phase}

The activities in this phase encompasses function specification, design and coding, testing and coordination of design. There may be configuration management processes for planning, ordering, providing and maintaining of software, hardware, and docware for the different test activities, on time and with good quality. During this phase, the project activities may be actively controlled and necessary actions taken to keep the project on time and on budget. The decision to continue may indicate revision of project and hand-over to customer and/or limited introduction on the market.

\subsection{Detailed analysis phase}

The purpose of this phase is to break up the outcomes of the project, evaluate the effectiveness of project organisation, compile the experiences gained, and suggest improvements and work models.

\subsection{The process model}

These phases are independent of whether the team is global or local. After analysing the functions, the business process of the design cycle is further analysed. The result of the analysis is a series of activities and sequences represented by IDEF3 model.

Each of the blocks in the IDEF3 model contains functional elements for carrying out GCE activities. For example, whiteboard is an essential element which to be supplied to the Project Concept phase. An agenda element will normally be included in Detailed Analysis phase. The system design is highly flexible to define these elements as add on components of the overall software so that minimum development costs can be achieved.

\section{CONCLUSION}

A system for Global Concurrent Engineering has been created from a study of the product development model. The system consists of three components each of which is designed to cater for specific aspects of a round the clock product design and development cycle. Further development of the system will continue along the current research and development direction. 


\section{REFERENCES}

Barkan, P., Hinckley, C.M. (1993) The Benefits and Limitations of Structured Design Methodologies. Manufacturing Review, 6(3), September, 211-220

Callaham, J.R. (1996) Preface of special issue. WET ICE'95 Fourth IEEE Workshop on Enabling Technologies: Infrastructure for Collaborative Enterprises, Computers in Industry, 29, 14.

Deitz, D. (1996) Engineering Online. Mechanical Engineering, September, 84-88. Dix, A. (1994) Computer Supported Cooperative Work: A Framework, in Design Issues in CSCW (ed. Rosenberg, D. and Hutchison, C.), Springer-Verlag, 9-26.

Dong, J., Parsaei, H.R., Leep, H.R. (1996) Manufacturing Process Planning in a Concurrent Design and Manufacturing Environment. Computers and Industrial Engineering, 30(1), 83-93.

Fichman, R.G., Kemerer, C.F. (1995) Object-Oriented and Conventional Analysis and Design Methodologies, in Readings in Object-Oriented Systems and Applications (ed. Rine, D.C.), 44-61

Fowler, S., Karinthi, R. (1996) Remote Access to CAD Database Using an Information Sharing System. Computers in Industry, 29, 117-122.

French, M.J. (1994) An Annotated List of Design Principles. Proc Instn Mech Engrs, 208(B4), 229-234

Frezza, S.T., Levitan, S.P., Chrysanthis, P.K. (1996) Linking Requirements and Design Data for Automated Functional Evaluation. Computers in Industry, 30, 13-25.

Kunz, J.C., Luiten, G.T., Fischer, M.A., Jin, Y., Levitt, R.E. (1996) CE4: Concurrent Engineering of Product, Process, Facility, and Organization. Concurrent Engineering: Research and Applications, 4(2), June, 187-198.

Love, T. (1996) New Developments in Engineering Design Theory, Pt.1 and 2. News Bulletin, IMechE Australian Branch, Iss.123, May, 13-17 and Iss.124, Nov, 8-13.

Marcos, A. (1996). Supporting Cooperative Software Development through a Multimedia Environment, in Multimedia/Hypermedia in Open Distributed Environments (ed. Herzner, W., Kappe, F.), Spring-Verlag, ISBN 3-21182587-8.

Nemes, L. and Bertok P. (1996) Cooperative Design on the Internet. IFIP World Congress on IT Tools, 2-6 September, Canberra, pp.419-428.

Prasad, B. (1996) Toward Definitions of a Concurrent Product design, Development, and Delivery (PD3) System. Concurrent Engineering: Research and Applications, 4(2), June, 102-109.

Sohlenius, G. (1992) Concurrent Engineering - Keynote Paper. Annals of the CIRP, 41(2), CIRP, 645-655. 


\section{BIOGRAPHY}

John Mo received his BSc in Mechanical Engineering and MSc in Industrial Engineering from the University of Hong Kong, and his $\mathrm{PhD}$ from Loughborough University of Technology, UK. He has 23 years of experience in industry and universities in various capacities including principal lecturer, laboratory manager and senior engineer. His research interests are in concurrent engineering, systems analysis and integration, process planning, real-time control and automation. He is a corporate member of a number of professional institutions and currently project manager at CSIRO.

Luigi Cirocoo received his BEng in Mechanical Engineering from the University of Adelaide, Australia. His research interests are in computer supported collaborative environment, holic manufacturing systems and scheduling. $\mathrm{He}$ is currently a research engineer in CSIRO.

Michael Kovacek is a computer officer in CSIRO responsible for the admininstration of PCs and network support. His research interests are in the integration and use of multimedia technologies to support various kinds of manufacturing activities. He is currently studying at the University of Melbourne in Computer Science. 